\title{
SYMMETRY AND ITS GENERALISATIONS
}

symmetrised components. The $h$ odd, $k$ odd reflections are associated with a doubly degenerate irreducible representation, offering a choice for the creation of two $C-1$ substructures that allowed identification of those parameter combinations worst determined and thus the best choice of constraints for optimising the refinement pathway.

Comparative refinement was used to show the correctness of the final structure solution and how appropriately chosen constrained refinement allowed escape from false minima.

Keywords: pseudo symmetry, twinning, constrained refinement

P.21.03.2

Acta Cryst. (2005). A61, C474

Relationships between Independent Molecules in $Z^{\prime}=\mathbf{2}$ Structures Anna Collins ${ }^{\mathrm{a}}$, David J. Watkin ${ }^{\mathrm{a}}$, Richard I. Cooper ${ }^{\mathrm{b}},{ }^{\mathrm{a}}$ Chemical Crystallography, University of Oxford, UK. ${ }^{\mathrm{b}}$ Oxford Diffraction, Abingdon, UK. E-mail: anna.collins@chem.ox.ac.uk

Approximately $11 \%$ of organic structures in the Cambridge Structural Database (CSD) [1] have more than one molecule in the asymmetric unit $\left(Z^{\prime}>1\right)$ and of these almost $90 \%$ have $Z^{\prime}=2$. Disorder in these structures is more common than in structures with $Z^{\prime}=1$ and the distribution of disorder between the crystallographicallyindependent molecules has been investigated.

A sizeable proportion of $Z^{\prime}=2$ structures are thought to exhibit pseudosymmetry. A method for assessing the extent of pseudosymmetry in $Z^{\prime}=2$ structures which do not exhibit disorder has been developed and suitable structures from the CSD have been evaluated using CRYSTALS [2].

Using this method, an analysis of the different relationships between molecules has been carried out. In particular, the nature of the rotation-translation operator relating the molecules in pseudosymmetric structures has been examined. The frequencies of different types of operator were considered for some common space groups, with emphasis on pseudo-translations and pseudo-inversions in non-centrosymmetric space groups which can pose particular problems in refinement [3]. This analysis is compared with previous work on the prevalence of certain symmetry elements [4].

[1] Allen F.H., Acta Cryst., 2002, B58, 380. [2] Betteridge P.W., Carruthers J.R., Cooper R.I., Prout K., Watkin D.J., J. Appl. Cryst., 2003, 36, 1487. [3] Marsh R.E., Acta Cryst., 1995, B51, 897. [4] Brock C.P., Dunitz J.D., Chem. Mater., 1994, 6, 1116

Keywords: pseudosymmetry, space-group symmetry, disorder

P.21.03.3

Acta Cryst. (2005). A61, C474

Twinning Investigation via the Primitive Cell of Bravais Lattices Giovanni Ferraris $^{\mathrm{a}}$, M. Nespolo ${ }^{\mathrm{b}}$, ${ }^{\mathrm{a}}$ Dpt. Sci. Mineral. Petrol., Univ. Torino; IGG - CNR, Italy. ${ }^{b} L C M^{3} B$, UMR - CNRS Univ. Henri Poincaré Nancy, France. E-mail: giovanni.ferraris@unito.it.

It is known that symmetry of the crystal structure lower than that of the corresponding Bravais lattice may trigger twinning [1]. Therefore, an analysis of the metric [2] symmetry of the Bravais lattice is a necessary step both to unravel real twins and to investigate the possibility of twinning.

The symmetry of a lattice is related to its metric properties; these can be investigated by different methods, like via metric tensor, equivalence of lattice vectors [3], occurrence of twofold axes [4], etc. Without pretension of originality, here we call attention on the importance of inspecting the metric of the primitive cells of the 7 centred Bravais lattices, which may in some cases be particularly remunerative.

(Pseudo) cubic and hR lattices - The primitive cells of $o I, t I, c F$ and $c I$ lattices always show $a=b=c$, a condition typical of cubic and $h R$ lattices. The latter occurs in $c F\left(\alpha=60^{\circ}\right)$ and $c I\left(\alpha=109.47^{\circ}\right)$ and is approached in $o I$ and $t I$ when $\alpha \cong \beta \cong \gamma$. The endemic $h P$ sublattice of an $h R$ lattice, which occurs also in $c P\left(\alpha=90^{\circ}\right)$, favours twinning by reticular (pseudo)merohedry.

(Pseudo) hP lattices - The primitive cells of $m C$ and $o C$ lattices have $a=b$, a condition which brings into existence an $h P$ (sub)lattice when $\gamma \cong 120^{\circ}$.
[1] Friedel G., Leçons de Cristallographie, Nancy, Berger - Levrault, 1926. [2] Nespolo M., Ferraris G., Z. Kristallogr., 2000, 215, 77. [3] Ferraris G., Ivaldi G., Acta Cryst., 1983, A39, 595. [4] Le Page Y., J. Appl. Cryst., 2002, 35, 175. Keywords: twinning, centred lattices, primitive lattices 